\title{
DEVELOPMENT AUTOMATED SYSTEM CONTROL HEAT CHAMBER BASED ON PROGRAMMABLE LOGIC CONTRO LER SIEMENS S7-200
}

\author{
Alexandr Nigay, Evgeny Kravchenko ${ }^{a}$ \\ ${ }^{1}$ National Research Tomsk Polytechnic University, 634050 Tomsk, Russia
}

\begin{abstract}
The aim of this work is to develop a computer-aided control and heat chamber on the basis of PLC Siemens S7-200, its installation, adjustment and subsequent commissioning.
\end{abstract}

\section{Introduction}

Systems for monitoring and control heat chamber consists of the following components:

1) Programmable logic controller S7 - 200;

2) Analog Input Module EM 231;

3) Normalizing converters $2000 \mathrm{~N}-22$;

4) The temperature sensor $50 \mathrm{Cu}$;

5) Emulator oven VC 10;

6) The relay $T 78$;

7) Test the capacity of the heat chamber;

8) Power Supply Sitop Smart.

\section{Functioning principle of the heat chamber}

The system of automated control heat chamber operates as follows: the pulse from the temperature sensor, which is disposed within the test container and measures the temperature of air supplied to the normalizing converter that has an output signal $4 \ldots 20 \mathrm{~mA}$. This signal is sent to the PLC, where it is compared with the setting and development of the control signal that is sent to the solenoid, which in turn, is the closure / open contacts ON / OFF control of the object - oven locomotive EP10 emulator.

The pulse from the temperature sensor, which is integrated directly into the heating element of the furnace emulator comes to normalizing converter koto-ing is the output signal of $4 \ldots 20 \mathrm{~mA}$. This signal is sent to the PLC, where it is compared with the alarm set point and produce the control signal which by-routed to an electromagnetic relay which in turn closes / opens the contacts on / off control of the object - oven locomotive EP10 emulator. The block diagram is shown in Figure 1.

${ }^{\text {a }}$ Corresponding author : kevatp@tpu.ru 


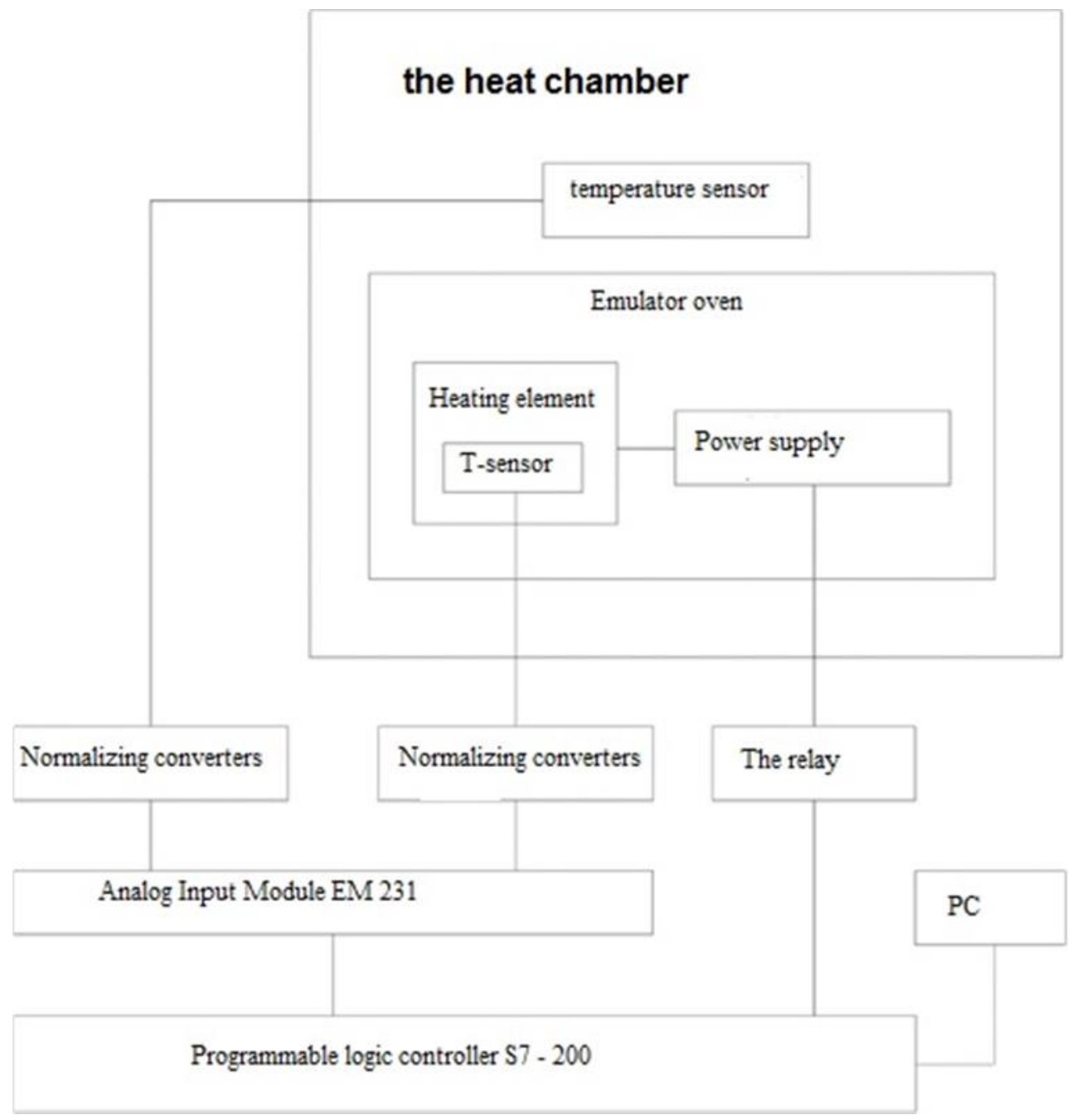

Figure 1. The block diagram of the control system and management of the heat chamber

\section{Development SCADA and system code}

The controller is programmed with a computer connected. Programming is done by the Step 7 - Micro / WIN. The program supports several programming languages: LAD (Ladder Diagram), STL (Statement List), FBD (Function Block Diagram). In the present work program has been developed in language LAD [1].

It is part of the program:

1) Implementation of scale-up procedures for non-compliance outputs analog transmitters (4 ... $20 \mathrm{~mA})$ and input analog input module $(0 \ldots 20 \mathrm{~mA})$;

2) compared with the settings;

3) Thermal protection (this protection is implemented to comply with the instructions for use of the emulator. Emergency setting is implemented in code and cannot be changed in the SCADA system, as opposed to the user settings;

4) SCADA system (Figure 2) -contains the operating and display unit, indicator temperature of the climate chamber and trends. 


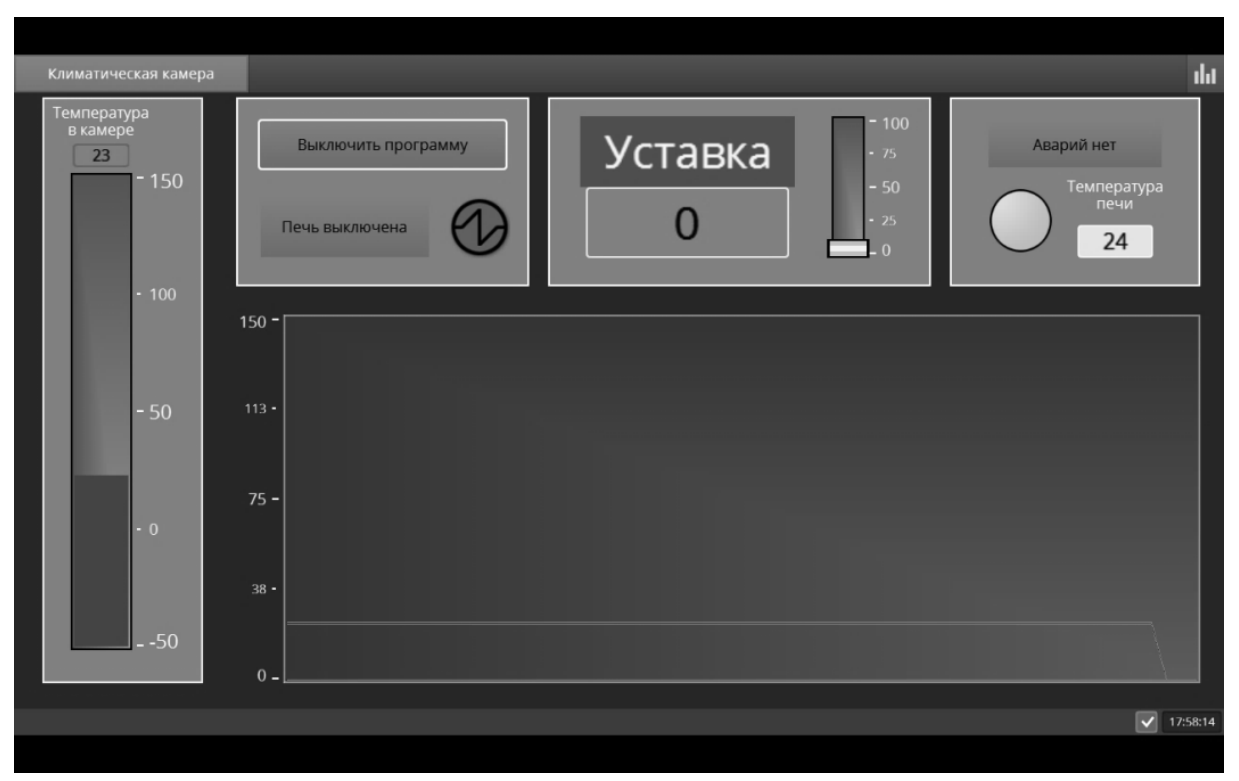

Figure 2. The SCADA system during operation

Developed SCADA system allows automation research in the field of diagnostics and reliability elements of automation systems [2,3] and power electronics [4,5].

\section{References}

1. Manual PLC S7-200.

2. G.V. Kuznetsov, E.V Kravchenko, Journal of Engineering Physics and Thermophysics, 80 (5), 1050 (2007)

3. E.C.W Jong, J.A Ferreira, P. Bauer, Power Electronics Letters, IEEE, 3, 125 (2005)

4. R. A Kurmangaliev, E. V. Kravchenko, EPJ Web of Conferences, 82, 01030 (2015)

5. E. V. Kravchenko, G. V Kuznetsov, MATEC Web Conf. 2014, 19, 01028 (2014)

6. E.V. Kravchenko, Kuznetsov G.V.,. EPJ Web of Conferences, 76 01014,(2014) 